\section{BSG GUIDELINE COMPLIANCE FOR THE MANAGEMENT OF ACUTE PANCREATITIS-ARE IMAGING DELAYS THE REAL ISSUE?}

R A J Spence, K McElvanna, I McAllister Surgical Unit, The Ulster Hospital, Belfast, Northern Ireland

\subsection{6/gutjnl-2013-305143.22}

Introduction The British Society of Gastroenterology (BSG) set standards for the management of acute pancreatitis; in particular, time until definitive treatment for gallstone pancreatitis.

Aims/Background We evaluated our management of acute pancreatitis against BSG guidelines, focusing on delays to definitive management.

Method Data were obtained retrospectively for 110 consecutive patients admitted with acute pancreatitis during a nine month period, and their management evaluated against the guidelines.

Results One hundred and ten patients (63 male, 47 female) were admitted with acute pancreatitis during a nine month period, with mean age 54.7 years. Aetiology included: gallstones (51 patients), alcohol (39), others (20). Ten patients $(9.1 \%)$ had severe pancreatitis, 6 of whom died. Overall mean length of stay: 5.8 days (range 2-78 days), gallstone pancreatitis 9.3 days, alcoholic pancreatitis 3.5 days, others 3.8 days. Mean wait for ultrasound: 1.7 days (range 0-5 days, with 59.4\% compliance with guidelines), MRCP, as inpatient 3.3 days (range 1-9 days), as outpatient 25 days (range 12-64 days). Intervention for gallstone pancreatitis included ERCP (13 patients; mean inpatient wait 6.4 days, outpatient 42 days); cholecystectomy (24 patients; mean wait: 12.5 days (range 1-81 days)). There was $75 \%$ compliance with BSG guidelines regarding definitive intervention for gallstone pancreatitis within 2 weeks.

Conclusion Delay for definitive intervention was partially attributable to imaging, especially as outpatient. Patients should be investigated as an inpatient to ensure guideline compliance with the 2 week rule for gallstone pancreatitis. ERCPs, either for stone removal, or as definitive management, must be performed as inpatient to avoid breach of guidelines. 